DOI:

УДК $672.11,539.3,536.7$

Н.Ю. Філоненко, к. ф.-м.н., с. н. с., natph2016@gmail.com

Інститут чорної металургії ім. 3.І. Некрасова НАН України (ІЧМ НАНУ) Дніпро

доцент, Дніпровський державний медичний університет, Дніпро

O.I. Бабаченко, д. т,. н., с. н. с., A_Babachenko@i.ua

директор Інституту чорної металургії ім. 3.І. Некрасова НАН України (ІЧМ НАНУ), Дніпро

Г.А. Кононенко, д. т. н., ст. д., perlit@ua.fm

вчений секретар Інституту чорної металургії ім. 3.І. Некрасова НАН України (ІЧМ НАНУ), Дніпро

\title{
МАТЕМАТИЧНЕ МОДЕЛЮВАННЯ СТІЙКОСТІ ПЕРВИННИХ ФАЗ ПРИ КРИСТАЛІЗАЦІЇ СПЛАВУ Fe-C-Mn-Si-Ti-Al-N
}

Після лиття в структурі сплавів Fe-C-Mn-Si-Ti-Al-N відбувається утворення дрібнодисперсних включень оксидів алюмінію $\mathrm{Al}_{2} \mathrm{O}_{3} \mathrm{ma}(\mathrm{Al}, \mathrm{Ti})_{2}(\mathrm{O}, \mathrm{N})_{3}$. Порівняння енергій Гіббса фаз $\mathrm{Al}_{2} \mathrm{O}_{3}$ та $(\mathrm{Al}, \mathrm{Ti})_{2}(\mathrm{O}, \mathrm{N})_{3}$, показало, що більш енергетично вигідне в сплаві утворення оксиду (Al, Ti) ${ }_{2}(\mathrm{O}, \mathrm{N})_{3}$, що узгоджується з експериментальними даними. Показано, що фаза $\mathrm{Al}_{2} \mathrm{O}_{3}$ термодинамічно стійка на всьому температурному інтервалі, а фаза $(\mathrm{Al}, \mathrm{Ti})_{2}(\mathrm{O}, \mathrm{N})_{3}$ втрачає свою термодинамічну стійкість при температурі $1423 \mathrm{~K}$.

Ключові слова: сталь; структурні компоненти; оксиди алюмінію; термодинамічні функиії.

After casting in the structure of alloys there is the formation of fine inclusions of aluminum oxides $\mathrm{Al}_{2} \mathrm{O}_{3}$ and $(\mathrm{Al}, \mathrm{Ti})_{2}(\mathrm{O}, \mathrm{N})_{3}$. The Gibbs energies for $\mathrm{Al}_{2} \mathrm{O}_{3}$ and $(\mathrm{Al}, \mathrm{Ti})_{2}(\mathrm{O}, \mathrm{N})_{3}$ phases are compared, and it is shown that the formation of $(\mathrm{Al}, \mathrm{Ti})_{2}(\mathrm{O}, \mathrm{N})_{3}$ oxide in the alloy is more energetically favorable, which is consistent with the experimental data. It is proved that the $\mathrm{Al}_{2} \mathrm{O}_{3}$ phase is thermodynamically stable over all the temperature range. The temperature dependence of determinant of thermodynamic stability of aluminum oxide $(\mathrm{Al}, \mathrm{Ti})_{2}(\mathrm{O}, \mathrm{N})_{3}$ has a distinct minimum at a temperature of $1423 \mathrm{~K}$.

Keywords: steel; structural components; aluminum oxides; thermodynamic functions.

\section{Постановка проблеми}

Актуальним завданням матеріалознавства $є$ дослідження, прогнозування та математичне моделювання умов утворення первинних фаз, дослідження їх термодинамічної стійкості та послідовності структурних перетворень у багатокомпонентних сплавах та сталях.

\section{Аналіз останніх досліджень та публікацій}

Для прогнозування утворення можливих фаз в багатокомпонентних сплавах та сталях використовують данні з діаграм станів бінарних та багатокомпонентних систем. Наразі проведені дослідження мікроструктури, фазового складу, як бінарних сплавів $\mathrm{Al}-\mathrm{Fe}, \mathrm{Fe}-\mathrm{Si}, \mathrm{Fe}-\mathrm{Ti}, \mathrm{Fe}-\mathrm{O}$ та інших, так і тернарних систем Al-Fe-Si, Al-Fe-Ti, Fe-Si-Ti [1-2]. Досить багато робіт присвячені дослідженню багатокомпонентних сплавів, їх фізичних та механічних властивостей [3-8].

Для прогнозування стабільності співіснуючих оксидних і нітридних фаз, що містять алюміній та титан були проаналізовані діаграми $\mathrm{Ti}-\mathrm{Al}-\mathrm{N}-\mathrm{O}, \mathrm{Ti}-\mathrm{Si}-\mathrm{N}-\mathrm{O}, \mathrm{Al}-\mathrm{Si}-\mathrm{N}-\mathrm{O}, \mathrm{Ti}-\mathrm{Al}-$ $\mathrm{Si}-\mathrm{N}, \mathrm{Al}-\mathrm{Fe}-\mathrm{O}-\mathrm{Si}$ та Ti-Al-Si-O $[4,9]$.

Кремній та алюміній мають вищу спорідненість до кисню, ніж Cr i Mn, тому вони більше впливають на утворення оксидів в сплавах [10].

Наразі в літературі відображені результати розрахунків термодинамічних функцій оксиду алюмінію [12-14], але в використаних методах не врахували флуктуаційні процеси.

\section{Формулювання мети досліджень}

Одним із важливих чинників утворення первинних фаз при кристалізації - енергетична складова. Отже, для визначення механізму утворення фаз необхідно виконати математичне моделювання термодинамічних функцій та термодинамічної стійкості оксидів алюмінію $\mathrm{Al}_{2} \mathrm{O}_{3}$ та 
$(\mathrm{Al}, \mathrm{Ti})_{2}(\mathrm{O}, \mathrm{N})_{3}$, їх залежності від температури за рівноважних та нерівноважних умов та виконати порівняльний аналіз.

\section{Виклад основного матеріалу}

Дослідження проводили на сплавах системи Fe-Mn-Si-Ti-Al-N-C 3 вмістом карбону

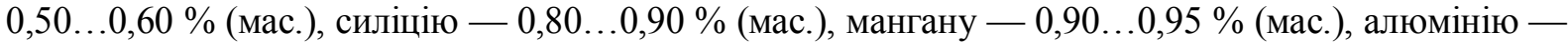
$0,20 \ldots 0,30 \%$ (мас.), титану - 0,02 ..0,03 \% (мас.), азоту - 0,015 ..0,02 \% (мас.) решта - залізо.

Виплавку сплаву проводили в печі в алундових тиглях в атмосфері аргону. Швидкість охолодження сплаву після лиття була $10 \mathrm{~K} / \mathrm{c}$. Металографічні шліфи сплаву виготовляли за стандартними методиками з застосуванням алмазних паст. Визначення хімічного складу сплаву було здійснене за допомогою хімічного та спектрального аналізів. Фазовий склад сплаву визначали на оптичному мікроскопі «Неофот- $21 »$. Результати мікрорентгеноспектрального аналізу були отримані за допомогою електронного мікроскопа JSM-6490 зі скануючою приставкою ASID-4D й енергодисперсійного рентгенівського мікроаналізатора «LinkSystems 860» із програмним забезпеченням. Для отримання фазового складу сплавів використали рентгеноструктурний аналіз на дифрактометрі ДРОН-3 у монохроматизованому $\mathrm{Fe}-\mathrm{K}_{\alpha}$ випромінюванні.

Мікроструктура сплаву Fe-Mn-Si-Ti-Al-N-Св литому стані була представлена перлітом, феритом та включеннями з різною морфологією (рис. 1, а).

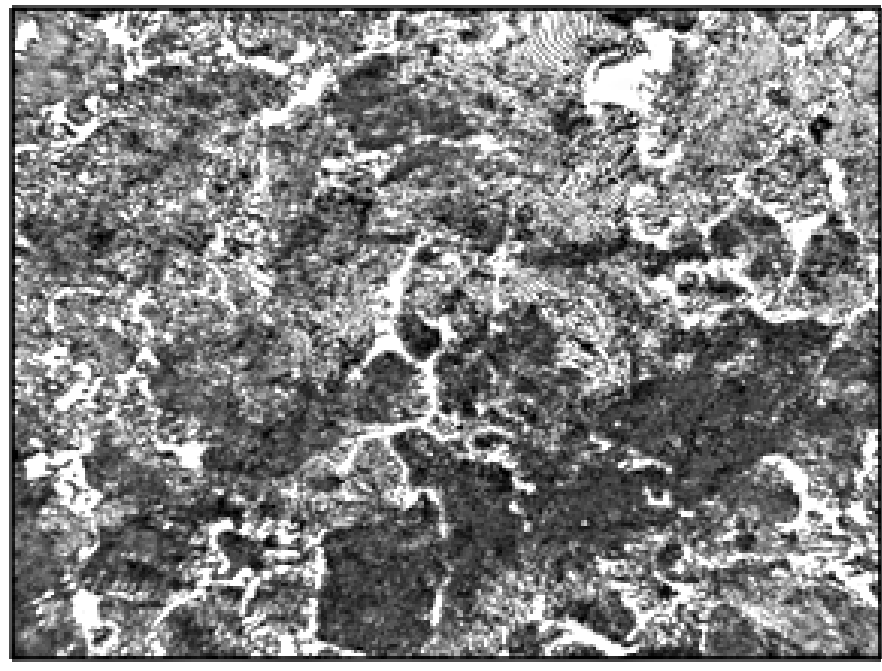

a

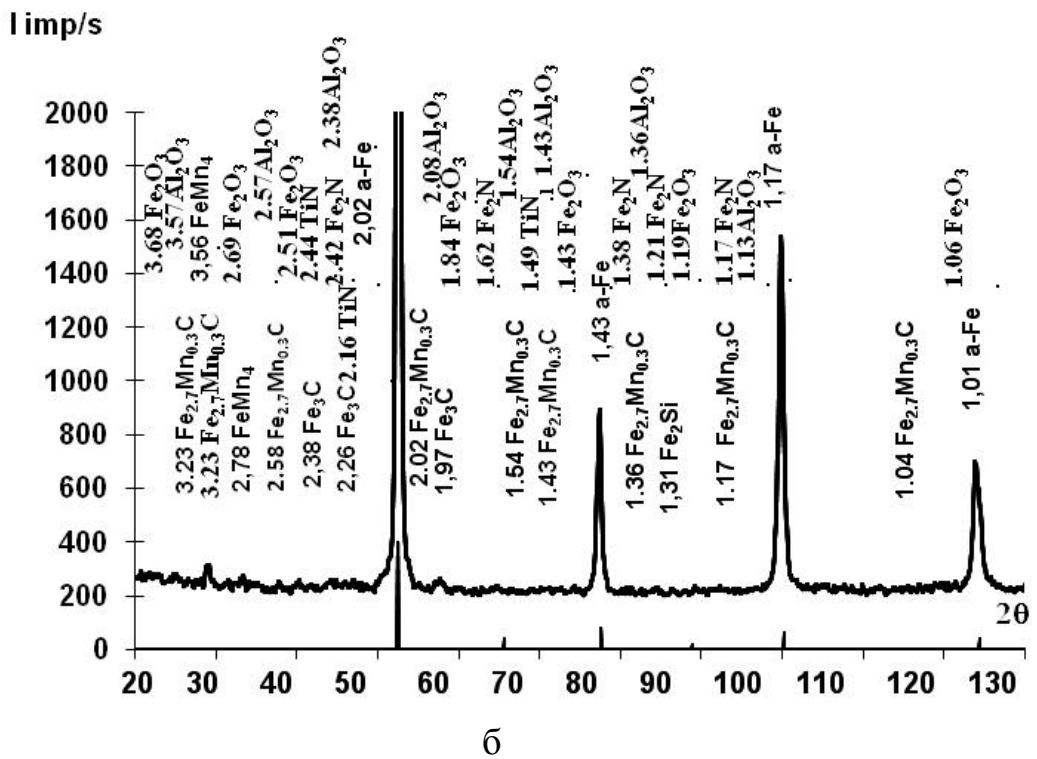

Puc. 1. Мікроструктура (а) та дифрактограма (б) сплаву системи Fe-CMn-Si-Ti-Al-N 
В структурі сплаву були виявлені первинні фази - окремі включення оксиду алюмінію $\mathrm{Al}_{2} \mathrm{O}_{3}$ та $(\mathrm{Al}, \mathrm{Ti})_{2}(\mathrm{O}, \mathrm{N})_{3}$, які утворились при кристалізації сплаву та мали розмір $1,5 \ldots 2$ мкм.

Для дослідження стійкості та термодинамічних функцій оксидів в даній роботі було виконане математичне моделювання.

Наразі в літературі відображені термодинамічні методи, для визначення термодинамічних функцій фаз, що не враховують внесок першого ступеня наближення високотемпературного розвинення термодинамічного потенціалу $[14,15]$.

\section{І. Енерхія Гіббса оксиду алюмінію}

При розрахунку енергії Гіббса оксиду алюмінію $\mathrm{Al}_{2} \mathrm{O}_{3} 3$ флуктуаційних процесів використали підхід [16, 17]

$$
G_{\mathrm{m}}^{\mathrm{Al}_{2} O_{3}}=y_{A l}{ }^{0} G_{A l}+y_{O}{ }^{0} G_{O}+R T\left(2 y_{A l} \ln y_{A l}+3 y_{O} \ln y_{O}\right)+y_{A l} y_{O} L_{A l: O}-\frac{L_{A l: O}^{2} y_{A l}^{2} y_{O}^{2}}{2 Z R T},
$$

де $G_{i}^{0}$ - енергія Гіббса хімічних елементів фази (Дж/моль), $R$ - універсальна газова стала ( $R=8,31$ Дж/(мольеК)), $T$ - температура (K), $L_{A l: O}$ - енергія взаємодії між компонентами в фазі (Дж/моль), $Z$ - координаційне число. Енергія взаємодії між елементами $L_{A l: O}$ залежить від температури. Температурну залежність енергії взаємодії між елементами представимо як: $L_{12}=a+b T+c T \ln T$.

Для мольних часток компонентів в сполуці чи сплаві виконується умова $\sum_{i=1}^{2} y_{i}=1$.

Для розрахунку енергії Гіббса оксиду алюмінію були використані енергії взаємодії між компонентами ${ }^{0} G_{A l},{ }^{0} G_{O}$ з роботи [18-19] та енергію $L_{A l: O}[16]$.

Отримано такий вираз для енергії Гіббса фази $\mathrm{Al}_{2} \mathrm{O}_{3}$

$$
G_{\mathrm{m}}^{\mathrm{Al}_{2} \mathrm{O}_{3}}=-45546,7+10,5 T+10^{5} T^{-1} .
$$

В літературі наведені вирази для енергії Гіббса оксиду алюмінію, які мають вигляд: $89,96+0,082 \mathrm{~T}, \mathrm{kJmol}^{-1}[20],-5512,92 \mathrm{~T}[11],-93660576,2 \mathrm{~T}^{-1}[16]$.

Таким чином, отримана залежність енергії Гіббса фази $\mathrm{Al}_{2} \mathrm{O}_{3}$ від температури 3 урахуванням флуктуацій дозволяє визначити ії значення в високотемпературній області, а також енергію Гіббса утворення даної фази при кристалізації.

\section{ІІ. Хімічний потенціал алюмінію та кисню в оксиді алюмінію}

Хімічний потенціал алюмінію було розраховано із застосуванням рівняння:

$$
\begin{gathered}
\mu_{A l}=\left(\frac{\partial G_{\mathrm{m}}^{\mathrm{Al}_{2} O_{3}}}{\partial y_{A l}}\right)_{T}={ }^{0} G_{A l}+R T 2\left(\ln y_{A l}+1\right)+y_{O} L_{A l: O}-\frac{L_{A l: O}{ }^{2}}{Z R T} y_{O}^{2} y_{A l} . \\
\mu_{A l}=-29895+0.6 T+5 \cdot 10^{5} T^{-1} .
\end{gathered}
$$

Хімічний потенціал кисню розрахували за наступною формулою

$$
\mu_{O}=-23756,7+3,2 T+6 \cdot 10^{5} T^{-1} .
$$

Якщо порівняти значення хімічних потенціалів алюмінію та кисню в оксиді алюмінію $\mathrm{Al}_{2} \mathrm{O}_{3}$, можна дійти висновку, що хімічний потенціал алюмінію більший, ніж кисню. Це дозволяє зробити припущення, що в фазі $\mathrm{Al}_{2} \mathrm{O}_{3}$ можливе заміщення атомів алюмінію атомами інших хімічних елементів, що спостерігали в даній роботі при утворенні багатофазних включень. Формування багатофазного включення починається з утворення з розплаву фази $\mathrm{L} \rightarrow \mathrm{AlTiO}_{3}$. Haвколо неї розміщена фаза $\left(\mathrm{Ti}_{0,3} \mathrm{Fe}_{0,2}\right)\left(\mathrm{N}_{0,3} \mathrm{C}_{0,2}\right)$, 3 стереометрією фази TiN. Крім цього, це дозволяє пояснити утворення твердих розчинів на базі фази $\mathrm{Al}_{2} \mathrm{O}_{3}[21]$ та фази $\mathrm{FeAlO}_{3}[11]$.

Як відомо з роботи [22], для визначення температури утворення фази $\mathrm{Al}_{2} \mathrm{O}_{3}$ необхідно знайти розв'язок рівняння

$$
\frac{\partial \mu}{\partial y}=0
$$


Хімічні потенціали алюмінію та кисню в оксиді алюмінію повинні мати мінімальні значення, які відповідають найбільш стабільному стану цієї фази

$$
\frac{\partial \mu_{A l}}{\partial y_{A l}}=\left(\frac{\partial^{2} G_{\mathrm{m}}^{\mathrm{Al}_{2} O_{3}}}{\partial y_{A l}^{2}}\right)_{T}=\frac{2 R T}{y_{A l}}-\frac{L_{A l: O}^{2} y_{O}^{2}}{Z R T}=0,\left(\frac{\partial^{2} G_{\mathrm{m}}^{\mathrm{Al}_{2} O_{3}}}{\partial y_{O}^{2}}\right)_{T}=\frac{3 R T}{y_{O}}-\frac{L_{A l: O}^{2} y_{A l}^{2}}{Z R T}=0 .
$$

Розв’язок рівнянь (2) дозволив знайти температуру утворення оксиду алюмінію $\mathrm{Al}_{2} \mathrm{O}_{3}-$ $T=1996,5$ К, що узгоджується $з$ результатами, наведеними у роботах $[11,16]$.

III. Ентропія, ентальпія та теплоємність оксиду алюмінію $\mathrm{Al}_{2} \mathrm{O}_{3}$

Ентропію фази $\mathrm{Al}_{2} \mathrm{O}_{3}$ визначили за формулою

$$
S=-\left(\frac{\partial G_{\mathrm{m}}^{\mathrm{Al}_{2} O_{3}}}{\partial T}\right)_{p}=-R\left(2 y_{A l} \ln y_{A l}+3 y_{O} \ln y_{O}\right)-\frac{L_{A l: O}^{2} y_{A l}^{2} y_{O}^{2}}{2 Z R T^{2}} .
$$

За результатами розрахунку ентропія оксиду алюмінію $\mathrm{Al}_{2} \mathrm{O}_{3}$ при температурі утворення дорівнює 270 Дж· моль ${ }^{-1} \mathrm{~K}^{-1}$, що добре узгоджується з результатами робіт $[16,18,23]$.

Для оксиду алюмінію $\mathrm{Al}_{2} \mathrm{O}_{3}$ було визначено теплоємність із застосуванням співвідно-

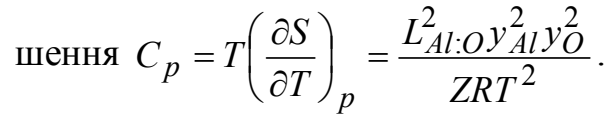

На рис. 2 представлено графічне відображення залежності теплоємності фази від температури.

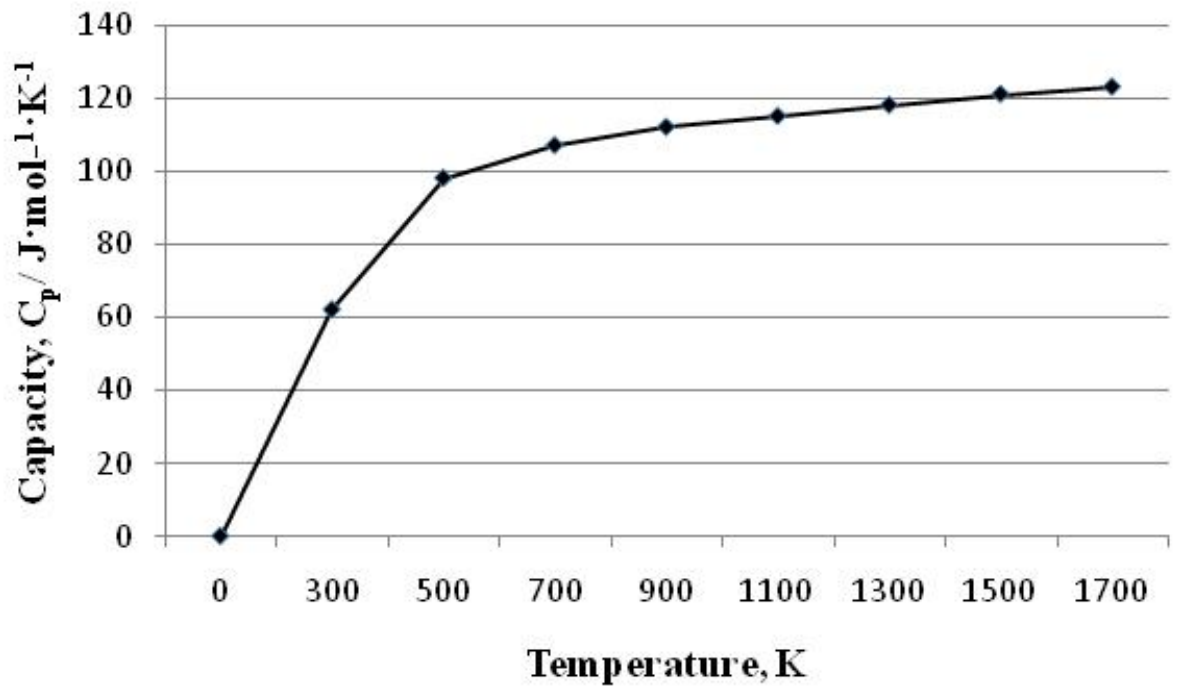

Puc. 2. Залежність теплоємності $\mathrm{C}_{\mathrm{p}}$ оксиду алюмінію $\mathrm{Al}_{2} \mathrm{O}_{3}$ від температури

Отримана залежність теплоємності $\mathrm{C}_{\mathrm{p}}$ від температури оксиду алюмінію $\mathrm{Al}_{2} \mathrm{O}_{3}$ дуже добре узгоджується з результатами наведеними в роботах $[16,23]$.

Таким чином, аналіз отриманих результатів дає змогу зробити висновок про те, що врахування внеску першого ступеня наближення високотемпературного розвинення термодинамічного потенціалу оксиду алюмінію $\mathrm{Al}_{2} \mathrm{O}_{3}$ дає можливість надати термодинамічний опис фази $\mathrm{Al}_{2} \mathrm{O}_{3} 3$ урахуванням флуктуаційних процесів, що необхідно враховувати при утворенні фази при кристалізації сплаву.

Рівняння температурної залежності енергій Гіббса з урахуванням флуктуацій в багатокомпонентних фазах отримали за формулою

$$
G_{\mathrm{m}}=\sum_{i=1}^{3} x_{i} G_{i}^{0}+R T \sum_{i=1}^{3} x_{i} \ln x_{i}+\frac{1}{2} \sum_{i} \sum_{i \neq \mathrm{j}} x_{i} x_{j} L_{i j}-\frac{1}{2} \sum_{i} \sum_{i \neq \mathrm{j}} \frac{L_{i j}^{2} x_{i}^{2} x_{j}^{2}}{2 Z T R} .
$$


Для отримання рівняння температурної залежності енергії Гіббса фази $(\mathrm{Al}, \mathrm{Ti})_{2}(\mathrm{O}, \mathrm{N})_{3}$ використали рівняння (3)

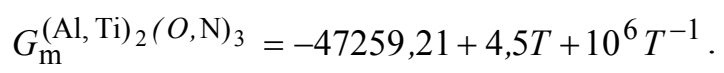

Проведені в даній роботі розрахунки енергія Гіббса фаз показали, що енергія Гіббса фази $(\mathrm{Al}, \mathrm{Ti})_{2}(\mathrm{O}, \mathrm{N})_{3}$ буде меншою, ніж фази $\mathrm{Al}_{2} \mathrm{O}_{3}$. Отриманий результат свідчить про те, що більш енергетично вигідно з розплаву утворення фази $(\mathrm{Al}, \mathrm{Ti})_{2}(\mathrm{O}, \mathrm{N})_{3}$, ніж оксиду $\mathrm{Al}_{2} \mathrm{O}_{3}$.

Одним із важливих факторів, що впливає на формування фаз при охолодженні є визначення ії термодинамічної стійкості, тобто температури, при якій вона спостерігається..

Енергія Гіббса фази, як відомо є функцією незалежних змінних $G=G(p, T, x)$, де $p-$ тиск, $T$ - температура, $x$ - масовий вміст елементів. За умов, що відсутня зовнішня сила та тиск не змінюється ( $p=c o n s t)$ повний диференціал енергії Гіббса має вид [24]

$$
d G=d U-d(T S)+d(p V)=-S d T+V d p+\mu d x .
$$

Відповідно термодинамічними силами $є S=-\left(\frac{\partial G}{\partial T}\right)_{x}$, а $\mu=\left(\frac{\partial G}{\partial x}\right)_{T}$, де $S-$ ентропія, $T$ - температура, $x$ - масова частка компонентів у сполуці. Для визначення стійкості фази знайдемо варіацію енергії Гіббса

$$
\delta G=\sum_{n=1}^{\infty} \frac{1}{n !}\left[\delta T \frac{\partial}{\partial T}+\delta x_{1} \frac{\partial}{\partial x_{1}}+\delta x_{2} \frac{\partial}{\partial x_{2}}\right]^{n} G .
$$

Загальна умова стійкості фази за Гіббсом полягає в тому, що в системі не повинні викликати як зворотні, так і не зворотні процеси (щоб система не вийшла з рівноваги), тому вони повинні бути таким, що

$$
\delta U-T \delta S+p \delta V-\mu \delta x>0,
$$

3 врахуванням співвідношення між внутрішньою енергією та вільною енергію Гіббса $U=G+T S-p V$, та того факту, що $\delta S=-\delta\left(\frac{\partial G}{\partial T}\right)_{x}=-\left(\frac{\partial \delta G}{\partial T}\right)_{u}, \delta \mu_{1}=-\left(\frac{\partial \delta G}{\partial x_{1}}\right)_{T}, \delta \mu_{2}=-\left(\frac{\partial \delta G}{\partial x_{2}}\right)_{T}$

Енергію Гіббса розвинемо в ряд за малими $\delta T$ та $\delta x$

$$
\begin{gathered}
\delta^{2} G=-\left(\frac{\partial G}{\partial T}\right)_{x} \delta T+\left(\frac{\partial G}{\partial x_{1}}\right)_{T} \delta x_{1}+\left(\frac{\partial G}{\partial x_{2}}\right)_{T} \delta x_{2}+\frac{1}{2}\left(\left(\frac{\partial^{2} G}{\partial T^{2}}\right)_{x} \delta T^{2}+2\left(\frac{\partial^{2} G}{\partial T \partial x_{1}}\right) \delta T \delta x_{1}+\right. \\
\left.+2\left(\frac{\partial^{2} G}{\partial T \partial x_{2}}\right) \delta T \delta x_{2}+\left(\frac{\partial^{2} G}{\partial x_{1}^{2}}\right)_{T} \delta x_{1}^{2}+\left(\frac{\partial^{2} G}{\partial x_{2}^{2}}\right)_{T} \delta x_{2}^{2}\right)+\ldots \\
\delta^{2} G=-S \delta T+\mu_{1} \delta x_{1}+\mu_{2} \delta x_{2}+\frac{1}{2}\left(\left(\frac{\partial S}{\partial T}\right)_{x} \delta T^{2}+2\left(\frac{\partial \mu_{1}}{\partial T}\right) \delta T \delta x_{1}+2\left(\frac{\partial \mu_{2}}{\partial T}\right) \delta T \delta x_{2}+\right. \\
\left.+\left(\frac{\partial \mu_{1}}{\partial x_{1}}\right)_{T} \delta x_{1}^{2}+\left(\frac{\partial \mu_{2}}{\partial x_{2}}\right)_{T} \delta x_{2}^{2}\right)+\ldots
\end{gathered}
$$

Для визначення похідних $\left(\frac{\partial \delta G}{\partial T}\right)_{\mathcal{u}},\left(\frac{\partial \delta G}{\partial x_{1}}\right)_{T}$ та $\left(\frac{\partial \delta G}{\partial x_{2}}\right)_{T}$ врахуємо лише члени першого i другого порядків 


$$
\begin{gathered}
\left(\frac{\partial \delta \mathrm{G}}{\partial T}\right)_{x}=-\left(\frac{\partial S}{\partial T}\right)_{x} \delta T+\left(\frac{\partial \mu}{\partial T}\right)_{x} \delta x+\frac{1}{2}\left[\left(\frac{\partial^{2} S}{\partial T^{2}}\right)_{x} \delta T^{2}+\left(\frac{\partial^{2} \mu}{\partial T \partial x}\right) \delta x^{2}+2\left(\frac{\partial^{2} \mu}{\partial T^{2}}\right)_{x} \delta x \delta T\right] \\
\left(\frac{\partial \delta \mathrm{G}}{\partial x_{1}}\right)_{T}=\left(\frac{\partial \mu_{1}}{\partial x_{1}}\right)_{T} \delta x+\left(\frac{\partial S}{\partial x_{1}}\right)_{T} \delta T+\frac{1}{2}\left[\left(\frac{\partial^{2} \mu_{1}}{\partial x_{1}^{2}}\right)_{T} \delta x^{2}+\left(\frac{\partial^{2} S}{\partial T \partial x_{1}}\right) \delta T^{2}+2\left(\frac{\partial^{2} \mu_{1}}{\partial T \delta x_{1}}\right)_{T} \delta x_{1} \delta T\right] \\
\left(\frac{\partial \delta \mathrm{G}}{\partial x_{2}}\right)_{T}=\left(\frac{\partial \mu_{2}}{\partial x_{2}}\right)_{T} \delta x+\left(\frac{\partial S}{\partial x_{2}}\right)_{T} \delta T+\frac{1}{2}\left[\left(\frac{\partial^{2} \mu_{2}}{\partial x_{2}^{2}}\right)_{T} \delta x_{2}^{2}+\left(\frac{\partial^{2} S}{\partial T \partial x_{2}}\right) \delta T^{2}+2\left(\frac{\partial^{2} \mu_{2}}{\partial T \delta x_{2}}\right)_{T} \delta x_{2} \delta T\right] .
\end{gathered}
$$

3 урахуванням, що за рівності змішаних похідних

$$
\left(\frac{\partial \delta \mathrm{G}}{\partial T}\right)_{x}=\left(\frac{\partial \delta \mathrm{G}}{\partial x}\right)_{T}=-\left(\frac{\partial S}{\partial x}\right)_{T}=\left(\frac{\partial \mu}{\partial T}\right)_{T},
$$

таким чином

$$
\begin{gathered}
\delta^{2} G=\frac{1}{2}\left(\left(\frac{\partial^{2} S}{\partial T^{2}}\right)_{x} \partial T^{2}+\left(\frac{\partial^{2} \mu_{1}}{\partial x_{1}^{2}}\right)_{T} \partial x_{1}^{2}+\left(\frac{\partial^{2} \mu_{2}}{\partial x_{2}^{2}}\right)_{T} \partial x_{2}^{2}+2\left(\frac{\partial \mu_{1}}{\partial T}\right)_{\mathrm{x}} \partial x_{1} \partial T+\right. \\
\left.+2\left(\frac{\partial \mu_{2}}{\partial T}\right)_{\mathrm{x}} \partial x_{2} \partial T+2\left(\frac{\partial^{2} \mu_{1}}{\partial x_{2}^{2}}\right)_{T} \partial x_{1} \partial x_{2}\right) .
\end{gathered}
$$

Якщо маємо квадратичну форму

$$
a_{11} x_{1}^{2}+a_{12} x_{1} x_{2}+a_{13} x_{1} x_{3}+\ldots+a_{n n} x_{n}^{2} \geq 0,
$$

то вона буде позитивною, коли будуть позитивними визначник, що складається з ії коефіцієнтів (критерій Сільвестра).

Таким чином, отримали матрицю

$$
D=\left|\begin{array}{lll}
\left(\frac{\partial S}{\partial T}\right)_{x_{1} x_{2}} & \left(\frac{\partial \mu_{2}}{\partial T}\right)_{x_{1} x_{2}} & \left(\frac{\partial \mu_{1}}{\partial T}\right)_{x_{1} x_{2}} \\
\left(\frac{\partial \mu_{2}}{\partial T}\right)_{x_{1} x_{2}} & \left(\frac{\partial \mu_{2}}{\partial x_{2}}\right)_{T x_{2}} & \left(\frac{\partial \mu_{2}}{\partial x_{1}}\right)_{T x_{1}} \\
\left(\frac{\partial \mu_{1}}{\partial T}\right)_{x_{1} x_{2}} & \left(\frac{\partial \mu_{1}}{\partial x_{2}}\right)_{T x_{2}} & \left(\frac{\partial \mu_{1}}{\partial x_{1}}\right)_{T x_{1}}
\end{array}\right| .
$$

Визначення термодинамічної рівноваги передбачає, що значення детермінанту D та коефіцієнти головної діагоналі повинні бути більше за нуль.

Випадок, коли $D=0$ був вперше зазначений Гіббсом, як критичний стан речовини. Геометричним місцем мінімумів детермінанту стійкості є лінія зниженої стійкості. Граничному випадку закритичних переходів відповідає максимальні значення флуктуацій, коли детермінант стійкості і коефіцієнти стійкості мають мінімальні значення. Визначимо значення, при яких $d D=0$.

Детермінант (4) з урахуванням (1) має вигляд

$$
\begin{gathered}
D=\left(-R\left(x_{1}\left(\ln x_{1}-1\right)-x_{2}\left(\ln x_{2}-1\right)-x_{1} x_{2}(b+c \ln T)+\right.\right. \\
\left.+\frac{(a+b T+c \ln T) x_{1}^{2} x_{2}^{2}(b+c \ln T)}{Z R T}-\frac{(a+b T+c T \ln T)^{2} x_{1}^{2} x_{2}^{2}}{2 Z R T^{2}}\right) . \\
\cdot\left(\frac{R T}{x_{2}}-\frac{(a+b T+c T \ln T)^{2} x_{1}^{2}}{Z R T} \cdot \frac{R T}{x_{1}}-\frac{(a+b T+c T \ln T)^{2} x_{2}^{2}}{Z R T}-\right.
\end{gathered}
$$




$$
\begin{aligned}
& \left.-\left((a+b T+c T \ln T)-\frac{2(a+b T+c T \ln T)^{2} x_{1} x_{2}}{Z R T}\right)^{2}\right)- \\
& -\left(R\left(\ln x_{2}+1\right)+x_{1}(b+c \ln T)+\frac{(a+b T+c T \ln T)^{2} x_{1}^{2} x_{2}}{Z R T^{2}}-\right. \\
& \left.-\frac{2(a+b T+c T \ln T) x_{1}^{2} x_{2}(b+c \ln T)}{Z R T}\right) \cdot\left(R\left(\ln x_{2}+1\right)+x_{1}(b+c \ln T)+\right. \\
& \left.+\frac{(a+b T+c T \ln T)^{2} x_{1}^{2} x_{2}}{Z R T^{2}}--\frac{2(a+b T+c T \ln T) x_{1}^{2} x_{2}(b+c \ln T)}{Z R T}\right) . \\
& \cdot\left(\frac{R T}{x_{1}}-\frac{(a+b T+c T \ln T)^{2} x_{2}^{2}}{Z R T}\right)-\left((a+b T+c T \ln T)-\frac{2(a+b T+c T \ln T)^{2} x_{1} x_{2}}{Z R T}\right) . \\
& \cdot\left(R\left(\ln x_{1}+1\right)+x_{2}(b+c \ln T)+\frac{(a+b T+c T \ln T)^{2} x_{1} x_{2}^{2}}{Z R T^{2}}-\right. \\
& \left.\left.-\frac{2(a+b T+c \ln T) x_{1} x_{2}^{2}(b+c \ln T)}{Z R T}\right)\right)+\left(R\left(\ln x_{1}+1\right)+x_{2}(b+c \ln T)+\right. \\
& \left.+\frac{(a+b T+c T \ln T)^{2} x_{1} x_{2}^{2}}{Z R T^{2}}-\frac{2(a+b T+c \ln T) x_{1} x_{2}^{2}(b+c \ln T)}{Z R T}\right) . \\
& \left.-\frac{2(a+b T+c T \ln T) x_{2} x_{1}^{2}(b+c \ln T)}{Z R T}\right) \cdot\left((a+b T+c T \ln T)-\frac{2(a+b T+c T \ln T)^{2} x_{1} x_{2}}{Z R T}\right)- \\
& -\left(R\left(\ln x_{1}+1\right)+x_{2}(b+c \ln T)+\frac{(a+b T+c T \ln T)^{2} x_{1} x_{2}^{2}}{Z R T^{2}}-\right. \\
& \left.\left.-\frac{2(a+b T+c T \ln T) x_{1} x_{2}^{2}(b+c \ln T)}{Z R T}\right) \cdot\left(\frac{R T}{x_{2}}-\frac{(a+b T+c T \ln T)^{2} x_{1}^{2}}{Z R T}\right)\right) .
\end{aligned}
$$

Умова виконується, коли

$$
\left(\frac{\partial D}{\partial T}\right)_{x}=0,\left(\frac{\partial D}{\partial x_{1}}\right)_{T}=0,\left(\frac{\partial D}{\partial x_{2}}\right)_{T}=0 .
$$

Отримана система рівнянь (5) була розв'язана в чисельному виді при використанні пакету Maple.

Результат розв'язку системи (5) для фаз $\mathrm{Al}_{2} \mathrm{O}_{3}$ та $(\mathrm{Al}, \mathrm{Ti})_{2}(\mathrm{O}, \mathrm{N})_{3}$ представлено на рис. 3. 


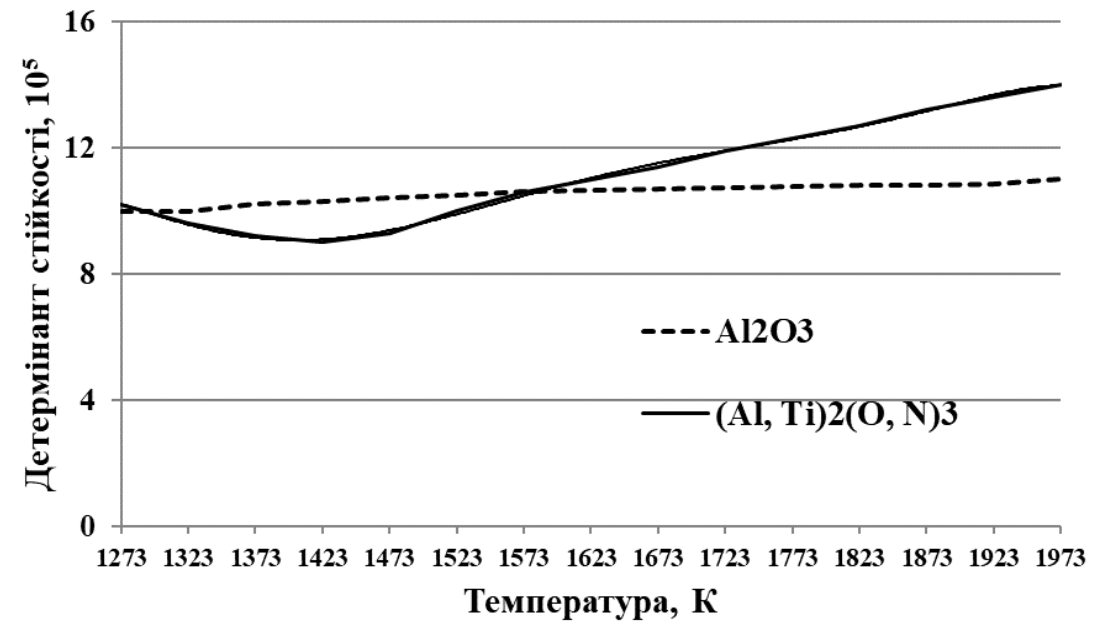

Puc. 3. Температурна залежність детермінанта стійкості фаз $\mathrm{Al}_{2} \mathrm{O}_{3}$ та $(\mathrm{Al}, \mathrm{Ti})_{2}(\mathrm{O}, \mathrm{N})_{3}$

Як бачимо з рис. 3, крива термодинамічної стійкості для фаз $\mathrm{Al}_{2} \mathrm{O}_{3}$ не має точок перегину. Це свідчить про те, що дана фаза є термодинамічно стабільною після кристалізації. Детермінант термодинамічної стабільності фази $(\mathrm{Al}, \mathrm{Ti})_{2}(\mathrm{O}, \mathrm{N})_{3}$ при 1423 К проходить через нечіткий мінімум. Падіння стабільності зі зниженням температури означає, що на мікроскопічному рівні дана фаза розпадається та починається утворення нової фази.

\section{Висновки}

1. У роботі досліджено структурні властивості сплаву Fe-Mn-Si-Ti-Al-N-C. Показано, що при кристалізації відбувається утворення окремих включень оксиду алюмінію $\mathrm{Al}_{2} \mathrm{O}_{3} \mathrm{Ta}(\mathrm{Al}$, $\mathrm{Ti})_{2}(\mathrm{O}, \mathrm{N})_{3}$.

2. Вперше виконані розрахунки енергії Гіббса, ентропії, теплоємності та визначена їх температурна залежність 3 врахуванням внеску першого ступеня наближення високотемпературного розвинення термодинамічного потенціалу оксиду алюмінію $\mathrm{Al}_{2} \mathrm{O}_{3}$. Слід зазначити, що відомі методи розрахунку термодинамічних функцій фаз не можуть бути використані для фаз в високотемпературній ділянці.

3. Запропонований в даній роботі термодинамічний підхід дав можливість визначити температуру утворення фази $\mathrm{Al}_{2} \mathrm{O}_{3}$, яка збігається з літературними даними.

4. Вперше виконано порівняння енергій Гіббса фаз $\mathrm{Al}_{2} \mathrm{O}_{3}$ та $(\mathrm{Al}, \mathrm{Ti})_{2}(\mathrm{O}, \mathrm{N})_{3}$, та показано, що більш енергетично вигідне в сплаві утворення оксиду $(\mathrm{Al}, \mathrm{Ti})_{2}(\mathrm{O}, \mathrm{N})_{3}$, що узгоджується 3 експериментальними даними.

5. Вперше показано, що фаза $\mathrm{Al}_{2} \mathrm{O}_{3}$ термодинамічно стійка на всьому температурному інтервалі. Температурна залежність детермінанту термодинамічної стійкості оксиду алюмінію $(\mathrm{Al}, \mathrm{Ti})_{2}(\mathrm{O}, \mathrm{N})_{3}$ має чіткий мінімум при температурі1423 К, що свідчить про те, що дана фаза втрачає свою термодинамічну стійкість, розпадається та починається утворення нової фази.

\section{Список використаної літератури}

1. Marker M. C.J., Duarte L. I., Leinenbach C., Richter K.W. Characterization of the Fe-rich corner of Al-Fe-Si-Ti. Intermetallics. 2013. № 39. C. 38-49.

2. Hidayat T., ShishinD., Jak E., Decterov S. Thermodynamic Reevaluation of the Fe-O System. Calphad. 2015. № 48. P. 131-144.

3. Tu Y., Huang L., Zhang Q., Zhou X., Jiang J. Effect of Si on the partitioning of Mn between cementite and ferrite, Materials Science and Technology. 2018. № 34. P. 780-785.

4. Caron M., Gagnon G., Fortin V., Currie J. F., Ouellet L., Tremblay Y., Biberger M., Reynolds R. Calculation of a Al-Ti-O-N quaternary isotherm diagram for the prediction of stable phases in TiN/Al alloy contact metallization. Journal of Applied Physics. 1996. Vol. 79. P. 4468-4478. 
5. Parusov E.V., Lutsenko V.A., ChuikoI. N., Parusov O.V. Influence of chemical composition and cooling parameters on kinetics of austenite decomposition in high-carbon steels. Chernye Metally. 2020. № 9. P. 39-44.

6. Lutsenko V.A., Parusov E.V., Golubenko T.N., Lutsenko O.V. Energy effective mode of softening heat treatment of silicon-manganese steel. Chernye Metally. 2019. № 11. P. 31-35.

7. Babachenko A.I., Togobitskaya D.N., Kononenko A.A., Snigura I.R., Kuksa O.V. Justification for Choosing Alloying and Micro-Alloying Elements to Improve the Mechanical Properties of Railway Wheels. Steel in Translation. 2020. № 50(11). P. 815 - 8217.

8. Babachenko A.I., Kononenko A.A. Influence of the chemical composition and structural state on tough properties of steel for railway wheels. Металофізики та новітні технологіï. 2008. № 30. P. 227-234.

9. Jak E., Hayes P.C. Thermodynamic Modelling of the Coal Ash Systems in BlackCoal Utilisation (a New FACT Database of the $\mathrm{SiO}_{2}-\mathrm{Al}_{2} \mathrm{O}_{3}-\mathrm{CaO}-\mathrm{FeO}-\mathrm{Fe}_{2} \mathrm{O}_{3}$ System and the Effects of $\mathrm{MgO}, \mathrm{K}_{2} \mathrm{O}$ and $\mathrm{Na}_{2} \mathrm{O}$ on Liquidus), in Proc.-Annu. Int. Pittsburgh Coal Conf. 2001. Vol. 18. P. 129-141.

10.Ciaś, A., Chemical Reactions During Sintering of Fe-Cr-Mn-Si-Ni-Mo-C Steels with Special Reference ti Processing in Semi-closed Containers. Science of Sintering. 2015. Vol. 47, No. 1.P. 61-69.

11.Prostakova V., Shishin D., Shevchenko M., Jak E. Thermodynamic optimization of the $\mathrm{Al}_{2} \mathrm{O}_{3}-$ $\mathrm{FeO}-\mathrm{Fe}_{2} \mathrm{O}_{3}-\mathrm{SiO}_{2}$ oxide system. CALPHAD: Computer Coupling of Phase Diagrams and Thermochemistry. 2019. Vol. 67. P. 101680(7).

12.Jung I., Eriksson G., Wu P., Pelton A. Thermodynamic Modeling of the $\mathrm{Al}_{2} \mathrm{O}_{3}-\mathrm{Ti}_{2} \mathrm{O}_{3}-\mathrm{TiO}_{2}$ SystemandIts Applications to the Fe-Al-Ti-O Inclusion Diagram. ISIJ International. 2009. Vol. 49(9). P. 1290-1297.

13.Furukawar G.T., Douglas T.B., McCoskeyr R.E., Ginnings D.C. Thermal Properties of Aluminum Oxide From $0^{\circ}$ to $1,200^{\circ}$ K. Journal of Research of the Nationa lBureau of Standards. 1956. 57(2). P. 2694-2700.

14.Bale C.W., Belisle E.P., Chartrand S.A., Decterov G., Eriksson A.E., Gheribi K., Hack I.H., Jung Y.B., Kang J., Melancon A.D., Pelton S., Petersen C., Robelin J., Sangster P., Spencer M.A. Fact Sage the chemicalss of ware and databases, 2010-2016. Calphad. 2016. Vol. 54. P. 35-53.

15.Decterov S.A. Termodynamic database for multicomponent oxide system. Chimica Techno Acta. 2018. №5(1). P. 16-48.

16.Filonenko N.Yu.. Structural state and thermodynamic stability of Al-Cu alloys. International Journal of Modern Physics B. 2020. Vol. 34, No. 8. 2050057 (11 pages).

17. Filonenko N.Yu., Galdina O.M., Kochenov A.V. Thermodynamic Functions of $\mathrm{Fe}^{3} \mathrm{~B}$ Borides. Physics and Chemistry of Solid State. 2019. № 20(2). P. 139-143.

18.Dinsdale T.A. SGTE dataforpureelements.Calphad.1991. №15(4). P. 317-425.

19. Mao H., Selleby M., Sundman, B. Phase Equilibria and Thermodynamics in the $\mathrm{Al}_{2} \mathrm{O} 3-\mathrm{SiO}_{2}$ System-Modeling of Mulliteand Liquid. J. Am. Ceram. Soc. 2005. № 88(9). P. 2544-2551.

20.Chen T., Gao M., Tong Y. Effects of Alloying Elements on the Formation of Core-Shell-Structured Reinforcing Particles during Heating of Al-Ti. Powder Compacts Materials. 2018. № 11(138). P. 2544-2551.

21.Escribano V. S., Amores J. M. G., Finocchio E., Daturib M., Busca G. Characterization of a(Fe,Al),O, Solid-solution Powders. J. Mater. Chem. 1995. Vol. 5(11). P. 1943-1951.

22.Базаров И. 3. Термодинамика. М.: Высшая школа, 1991 - 376 с.

23.Erba A., Mau J., Demichelisc R., Dovesia R. Assessing thermochemical properties of materials through hinitio quantum-mechanical methods: thecaseofa- $\mathrm{Al}_{2} \mathrm{O}_{3}$. Phys. Chem. Chem. Phys. 2015. Vol. 17. P. 11670-11677.

24.Filonenko N.Yu.,Galdina O.M. Liquidus surface and spinodal of Fe-B-C alloys. East European Journal of Physics. 2020. № 1. P. 75-82. 


\title{
MATHEMATICAL MODELING OF PRIMARY PHASES STABILITY DURING CRYSTALLIZATION OF Fe-C-Mn-Si-Ti-Al-N ALLOY Filonenko N., Babachenko O., Kononenko H.
}

\begin{abstract}
The crucial task of materials science is investigation, forecasting and mathematical modeling of the conditions of primary phases formation, the study of their thermodynamic stability and the structural transformations sequence in multicomponent alloys and steels.

The object of this paper is to investigate the thermodynamic functions and thermodynamic stability of aluminum oxides $\mathrm{Al}_{2} \mathrm{O}_{3}$ and $(\mathrm{Al}, \mathrm{Ti})_{2}(\mathrm{O}, \mathrm{N})_{3}$, their dependence on temperaturewith accounting for the first degree of approximation of high-temperature expansion for thermodynamic potential of binary alloys, perform a comparative analysis.

The structural properties of the Fe-Mn-Si-Ti-Al-N-C alloy are considered in this work. It is shown that under crystallization the formation of separate inclusions of aluminum oxides $\mathrm{Al}_{2} \mathrm{O}_{3}$ and $(\mathrm{Al}, \mathrm{Ti})_{2}(\mathrm{O}, \mathrm{N})_{3}$ occurs.

For the first time, calculations of Gibbs energy, entropy, heat capacity are performed and their dependence on temperature is derived taking into account the contribution of the first degree of approximation for high-temperature expansion of thermodynamic potential of aluminum oxide $\mathrm{Al}_{2} \mathrm{O}_{3}$. It should be noted that the well-known methods for calculating the thermodynamic functions of the phases can be used only under equilibrium conditions and do not take into account fluctuation processes. The method proposed in this paper makes it possible to determine the temperature of formation of the $\mathrm{Al}_{2} \mathrm{O}_{3}$ phase, which coincides with data of other authors. The Gibbs energies for $\mathrm{Al}_{2} \mathrm{O}_{3}$ and $(\mathrm{Al}, \mathrm{Ti})_{2}(\mathrm{O}, \mathrm{N})_{3}$ phases are compared, and it is shown that the formation of $(\mathrm{Al}, \mathrm{Ti})_{2}(\mathrm{O}, \mathrm{N})_{3}$ oxide in the alloy is more energetically favorable, which is consistent with the experimental data. It is proved that the $\mathrm{Al}_{2} \mathrm{O}_{3}$ phase is thermodynamically stable over all the temperature range. The temperature dependence of determinant of thermodynamic stability of aluminum oxide $(\mathrm{Al}, \mathrm{Ti})_{2}(\mathrm{O}, \mathrm{N})_{3}$ has a distinct minimum at a temperature of $1423 \mathrm{~K}$, which indicates that this phase loses its thermodynamic stability, decomposes and begins to form a new phase.

Mathematical modeling of thermodynamic functions of phases provided in this work can be applied further to any alloys and steels.
\end{abstract}

\section{References}

[1] Marker M C.J., Duarte L.I.., Leinenbach C., Richter K.W. (2013). Characterization of the Fe-rich corner of Al-Fe-Si-Ti. Intermetallics, 39, 38-49. [in English]

[2] Hidayat T., Shishin D., Jak E., Decterov S. (2015). Thermodynamic Reevaluation of the Fe-O System. Calphad, 48, 131-144. [in English]

[3] Tu Y., Huang L., Zhang Q., Zhou X., Jiang J. (2018). Effect of Sion the partitioning of Mn between cementite and ferrite. Materials Scienceand Technology. 34(7), 780-785. [in English]

[4] Caron M., Gagnon G.,Fortin V., Currie J. F., Ouellet L., Tremblay Y., Biberger M., Reynolds R. (1996). Calculation of a Al-Ti-O-N quaternary isotherm diagram for the prediction of stable phases in TiN/Al alloy contact metallization. Journal of Applied Physics, 79, 4468-4478. [in English]

[5] Parusov E. V., Lutsenko V. A., Chuiko I. N., Parusov O. V. (2020). Influence of chemical composition and cooling parameters on kinetics of austenite decomposition in high-carbon steels.. Chernye Metally, 9, P. 39-44. [in English]

[6] Lutsenko V.A., Parusov E.V., Golubenko T.N., Lutsenko O.V. (2019). Energy effective mode of softening heat treatment of silicon-manganese steel. Chernye Metally,. 11, 31-35. [in English]

[7] Babachenko A.I., Togobitskaya D.N., Kononenko A.A., Snigura I.R., Kuksa O.V. (2020). Justification for Choosing Alloying and Micro-Alloying Elements to Improve the Mechanical Properties of Railway Wheels. Steelin Translation, 50(11), 815-817. [in English]

[8] Babachenko A.I., Kononenko A.A. (2008.). Influence of the chemical composition and structural state on tough properties of steel for railway wheels. Metallofizika i Noveishie Tekhnologii Metalphysics and the latest technologies, 30, 227-234. [in English] 
[9] Jak E., Hayes P.C. (2001). Thermodynamic Modelling of the Coal Ash Systems in BlackCoal Utilisation (a New FACT Database of the $\mathrm{SiO}_{2}-\mathrm{Al}_{2} \mathrm{O}_{3}-\mathrm{CaO}-\mathrm{FeO}-\mathrm{Fe}_{2} \mathrm{O}_{3}$ System and the Effects of $\mathrm{MgO}, \mathrm{K}_{2} \mathrm{O}$ and $\mathrm{Na}_{2} \mathrm{O}$ on Liquidus), in Proc.-Annu. Int. Pittsburgh Coal Conf. 2001. Vol. 18. P. 129-141. [in English]

[10] Ciaś, A. (2015). Chemical Reactions During Sintering of Fe-Cr-Mn-Si-Ni-Mo-C Steels with Special Reference ti Processing in Semi-closed Containers. Science of Sintering, 47(1), 61-69. [in English]

[11] Prostakova V., Shishin D., Shevchenko M., Jak E. (2019). Thermodynamic optimization of the $\mathrm{Al}_{2} \mathrm{O}_{3}-\mathrm{FeO}-\mathrm{Fe}_{2} \mathrm{O}_{3}-\mathrm{SiO}_{2}$ oxide system. CALPHAD: Computer Coupling of Phase Diagrams and Thermochemistry, 67, 101680(7). [in English]

[12] JungI., ErikssonG., Wu,P.,PeltonA. (2009). Thermodynamic Modeling of the $\mathrm{Al}_{2} \mathrm{O}_{3}-\mathrm{Ti}_{2} \mathrm{O}_{3}-$ $\mathrm{TiO}_{2}$ SystemandIts Applications to the $\mathrm{Fe}-\mathrm{Al}-\mathrm{Ti}-\mathrm{O}$ Inclusion Diagram. ISIJ International, 49(9), 1290-1297. [in English]

[13] Furukawar G.T., Douglas T.B., McCoskeyr R.E., Ginnings D.C (1956). Thermal Properties of Aluminum Oxide From $0^{\circ}$ to $1,200^{\circ} \mathrm{K}$. Journal of Research of the National Bureau of Standards, 57(2), 2694-2700. [in English]

[14] Bale C.W., Belisle E.P., Chartrand S.A., Decterov G., Eriksson A.E., Gheribi K., Hack I.H., Jung Y.B., Kang J., Melancon A.D., Pelton S., Petersen C., Robelin J., Sangster P., Spencer M.A. (2016). Fact Sage the chemicalss of ware and databases, 2010-2016. Calphad, 54, 35-53. [in English]

[15] Decterov S.A. (2018). Termodynamic database for multicomponent oxide system Chimica Techno Acta, 5(1), 16-48. [in English]

[16] Filonenko N.Yu.. (2020). Structural state and thermodynamic stability of Al-Cu alloys. International Journal of Modern Physics B, 34(8), 2050057 (11). [in English]

[17] Filonenko N. Yu., Galdina O.M., Kochenov A.V. (2019). Thermodynamic Functions of $\mathrm{Fe}_{3} \mathrm{~B}$ Borides. Physics and Chemistry of Solid State, 20(2), 139-143. [in English]

[18] Dinsdale T.A. (1991). SGTE dataforpureelements. Calphad, 15(4), 317-425. [in English]

[19] Mao H. ,Selleby M., Sundman B. (2005). Phase Equilibria and Thermodynamics in the $\mathrm{Al}_{2} \mathrm{O} 3-$ $\mathrm{SiO}_{2}$ System-Modeling of Mulliteand Liquid. J. Am. Ceram. Soc, 88 (9), 2544-2551. [in English]

[20] Chen T., Gao M., Tong Y. (2018 Effects of Alloying Elements on the Formation of Core-ShellStructured Reinforcing Particles during Heating of Al-Ti. Powder Compacts Materials, 11(138), 2544-2551. [in English]

[21] Escribano V.S., Amores J. M. G., Finocchio E., Daturib M., Busca G. (1995). Characterization of a-(Fe,Al),O, Solid-solution Powders. J. Mater. Chem, 5(11), 1943-1951. [in English]

[22] Bazarov I.P. (1991). Termodinamika [Thermodynamics]. M: Vichashkola [In Russian]

[23] Erba A., Mau J., Demichelisc R., Dovesia R. (2015). Assessing thermochemical properties of materials through hinitio quantum-mechanical methods: thecaseofa- $\mathrm{Al}_{2} \mathrm{O}_{3}$. Phys. Chem. Chem. Phys, 17, 11670-11677. [in English]

[24] Filonenko N.Yu., Galdina O.M. (2020). Liquidus surface and spinodal of Fe-B-C alloys. East European Journal of Physics, 1, 75-82. [in English] 